\title{
Y-Shaped Intra-Scleral Fixated Lens versus Retro-Pupillary Iris Claw Lens in the Treatment of Aphakia
}

\author{
Ahmed Mohamed Morshed (), Mahmoud Nasr Aldeeb, Adel Kamal Abdeen, \\ Salah Mohamed Almosalamy
}

Ophthalmology Department, Faculty of Medicine, Zagazig University, Zagazig, Egypt

Email:ahmed_morshed2017@yahoo.com

How to cite this paper: Morshed, A.M., Aldeeb, M.N., Abdeen, A.K. and Almosalamy, S.M. (2019) Y-Shaped Intra-Scleral Fixated Lens versus Retro-Pupillary Iris Claw Lens in the Treatment of Aphakia. Open Journal of Ophthalmology, 9, 105-124. https://doi.org/10.4236/ojoph.2019.92012

Received: March 29, 2019

Accepted: May 27, 2019

Published: May 30, 2019

\section{Copyright $\odot 2019$ by author(s) and} Scientific Research Publishing Inc. This work is licensed under the Creative Commons Attribution International License (CC BY 4.0).

http://creativecommons.org/licenses/by/4.0/

\begin{abstract}
Background: Many reasons can lead to an aphakia without adequate capsular support for implantation of a posterior chamber intraocular lens (IOL), such as intraoperative unintentional rupture of posterior capsule during phacoemulsification, planned intracapsular cataract extraction, ocular trauma and lens dislocation due to congenital and acquired causes. Purpose: To compare Y-shaped intra-scleral fixation of a posterior chamber IOL with retro pupillary fixation of an iris-claw intraocular lens (IOL) for Aphakic eyes without sufficient capsular support as respects safety, visual recovery and complications of both methods. Patients and Methods: One hundred Aphakic eyes were arbitrarily distributed between two groups. Group A included 50 eyes treated with retropupillary fixation of iris claw lens and group B included 50 eyes treated with Y-shaped intra-scleral fixation technique. Preoperative, intraoperative and postoperative data were analysed including best corrected visual acuity (BCVA), intraocular pressure (IOP), surgical time, intraoperative problems, IOL malposition and postoperative complications. Following up on patients was carried out for at least six months. Results: The mean duration of surgery was $21 \pm 5.3 \mathrm{~min}$ in group $\mathrm{A}$ and was $53.4 \pm 6.9 \mathrm{~min}$ in group B (p-value $<0.001$ ). After 6 months, the mean BCVA was $0.34 \pm 0.15$ in group $A$ and was $0.31 \pm 0.16$ in group $B(p>0.05)$. IOL tilt was found in 0 $(0 \%)$ eyes in group $A$ and in $5(10 \%)$ eyes in group $B(p<0.05)$. IOL decentration was observed in $1(2 \%)$ eye in group A and $7(14 \%)$ eyes in group B, with statistically significant difference ( $\mathrm{p}$ value $=0.03$ ). Cystoid macular edema was found in $2(4 \%)$ eyes in group A and in $5(10 \%)$ eyes in group B ( $p>$ $0.05)$. Conclusion: The results of our study indicated that both methods are satisfactory in correcting aphakia without sufficient capsular support as regards postoperative best corrected visual acuity (BCVA); however the surgical
\end{abstract}


technique of retropupillary iris claw lens is easier, shorter, with low intra- and postoperative complications and safer than those used for intra-scleral fixation of IOL. But for eyes which lack both iris and capsular support, a scleral fixation of a posterior chamber IOL remains the only option.

\section{Keywords}

Aphakia, Iris-Claw Intraocular Lens (IOL), Y-Shaped Intra Scleral Fixation

\section{Introduction}

In the absence of enough capsular support, the best choice for an IOL implantation remains a difficult issue. Different intraocular lenses (IOLs) including angle supported anterior chamber intraocular lenses (ACIOLs), scleral fixation posterior chamber intraocular lenses (SF-PCIOL) and iris claw anterior chamber intraocular lenses (IC-ACIOLs) have been used to correct aphakia [1]. Angle-supported anterior chamber IOL has many advantages such as ease of insertion, decreased intraoperative time and minimal intra-operative manipulations. However, a number of problems such as progressive damage to corneal endothelial cells, persistent inflammation in the anterior chamber, secondary glaucoma, and cystoid macular edema have been reported [2].

Sutured SF-PCIOL implantation is technically difficult, time-consuming and is associated with many problems such as IOL tilt, decentration and sutures erosion through the conjunctiva; so some surgeons attempted implantation of iris claw IOL, but this resulted in some problems such as recurrent iritis, pupillary block, and IOL instability. Also technique of iris claw IOL implantation is not suitable for patients with iris defects such as traumatic iridectomy or Iridodialysis [3]. For the above reasons intra-scleral fixation approach has been added to the recent cataract surgery practice [4]. The aim of this work is to compare retro pupillary fixation of an iris-claw intraocular lens (IOL) with Y-shaped intra-scleral fixation of a posterior chamber IOL for Aphakic eyes without sufficient capsular support as regards visual recovery, safety and complications of both procedures.

\section{Patients and Methods}

This is a prospective interventional case series with a hundred eyes of ninety six patients, chosen from those attending the ophthalmology department at Zagazig University hospitals between February 2016 and May 2018, according to the inclusion and exclusion criteria.

Inclusion criteria: aphakia with insufficient capsular support, with best corrected visual acuity (BCVA) 4/60 or better (except cases presented with anterior dislocation of crystalline lens) including: cases of phacoemulsification with intra-operative unintentional rupture of posterior capsule with insufficient sup- 
port for IOL implantation, planned I.C.C.E due to sever lens subluxation e.g. pseudoexofoliation syndrome and cases of traumatic cataract resulted from globe rupture with sever posterior capsule tears, not implanted at primary interference.

Exclusion criteria: aphakia with decompensated corneas, inadequately iris tissue, uncontrolled glaucoma, persistent or recurrent uveitis, posterior segment pathology and in patient with no useful vision in the fellow eye.

Complete ophthalmic examinations were performed to all patients including. (A) Visual acuity: The BCVA was measured. Refraction is made using Topcon KR-8900 autorefractokeratometer (Topcon Corporation, Tokyo, Japan). (B) Slit lamp bio microscopy of the anterior segment. (C) Fundus examination (D) Tonometry: The intra ocular pressure was measured preoperatively and postoperative using Goldman applanation tonometer. (E) Keratometric readings using Topcon KR-8900 autokerato refractometer (Topcon Corporation, Tokyo, Japan). (F) Specular microscopy (NIDEK CEM-530) was done to assess the endothelial cell status. (G) white-to-white (WTW) diameter was measured by ZEISS IOL-Master (Carl Zeiss Meditec, Jena, Germany) before the surgery to identify the amount of haptic availability for tucking after haptic externalization in cases with Y-shaped intra scleral fixated IOL. (H) fully informed consent was obtained from all patients.

The IOL implantation was done either as a primary procedure or as a secondary procedure after at least $3-4$ weeks from the primary procedure. Most of cases were performed under local anesthesia (peribulbar injection of mixture of lignocaine, bupivacaine and hylase) unless there was indication for general anesthesia e.g. young age; all eyes were operated upon by the same surgeon. The one hundred eyes were divided randomly into 2 groups (A and $\mathrm{B}$ ) according to their order of presentation. Group A: included fifty eyes in which the iris claw IOL was implanted, the lens used in this work was the Artisan iris claw aphakia IOL (Ophtec BV, Groningen, The Netherlands) which is a PMMA IOL with an $8.5 \mathrm{~mm}$ length, $1.04 \mathrm{~mm}$ maximum height and $5.4 \mathrm{~mm}$ optical zone width. The manufacturer's recommended A-constant of 116.8 with the Sanders-Retzlaff-Kraff II formula to calculate the IOL power for retropupillary implantation. Group B: included fifty eyes in which a 3-Piece monofocal IOL was used, the lens used in this study was (Tecnis ZA9003; Abbott Medical Optics, Santa Ana, CA). The IOL is $6 \mathrm{~mm}$ UV-blocking hydrophobic acrylic biconvex optic with OptiEdge design; $13 \mathrm{~mm}$ overall diameter. The manufacturer's recommended A-constant 119.

Technique of surgery: In retropupillary iris claw group, two side ports were done; anterior vitrectomy was performed when required. A superior $5.5 \mathrm{~mm}$ clear corneal incision was made. Sodium hyaluronate 1.0\% Healon GV (Abbott, USA) was injected through the primary incision to inflate and maintain the anterior chamber. Iris claw IOL was introduced into the anterior chamber through the corneal incision upside down, in a reversed position (convex side down); ro- 
tating the lens such that haptics were in line with the side ports. The IOL optic was held with a lens forceps, one haptic was pushed beneath the iris. Simultaneously, dialor was introduced through the paracentesis on the same side and enclavation done. End point was noting the dimple at the site of enclavation which indicate engagement of adequate iris tissue. Similarly, haptic enclavation in the other side was performed. Closure of the clear corneal incision by 10-0 nylon sutures (Figure 1).

In intra-Scleral fixation group, conjunctival peritomy was made at the 2 o'clock and 8 o'clock positions. $2 \mathrm{Y}$-shaped incisions were done $1.5 \mathrm{~mm}$ from the limbus precisely 180 degrees apart diagonally. Anterior vitrectomy was performed when required. Sclerotomy is done parallel to the iris at the Y-shaped incision with a 23-gauge MVR knife and a scleral tunnel was made parallel to the limbus at the branching point of the Y-shaped incision. A 2.4 to $3.0 \mathrm{~mm}$ keratome was utilized to form a corneal incision at 10 o'clock through which a standard 3-piece monofocal IOL is implanted with an injector; the trailing haptic
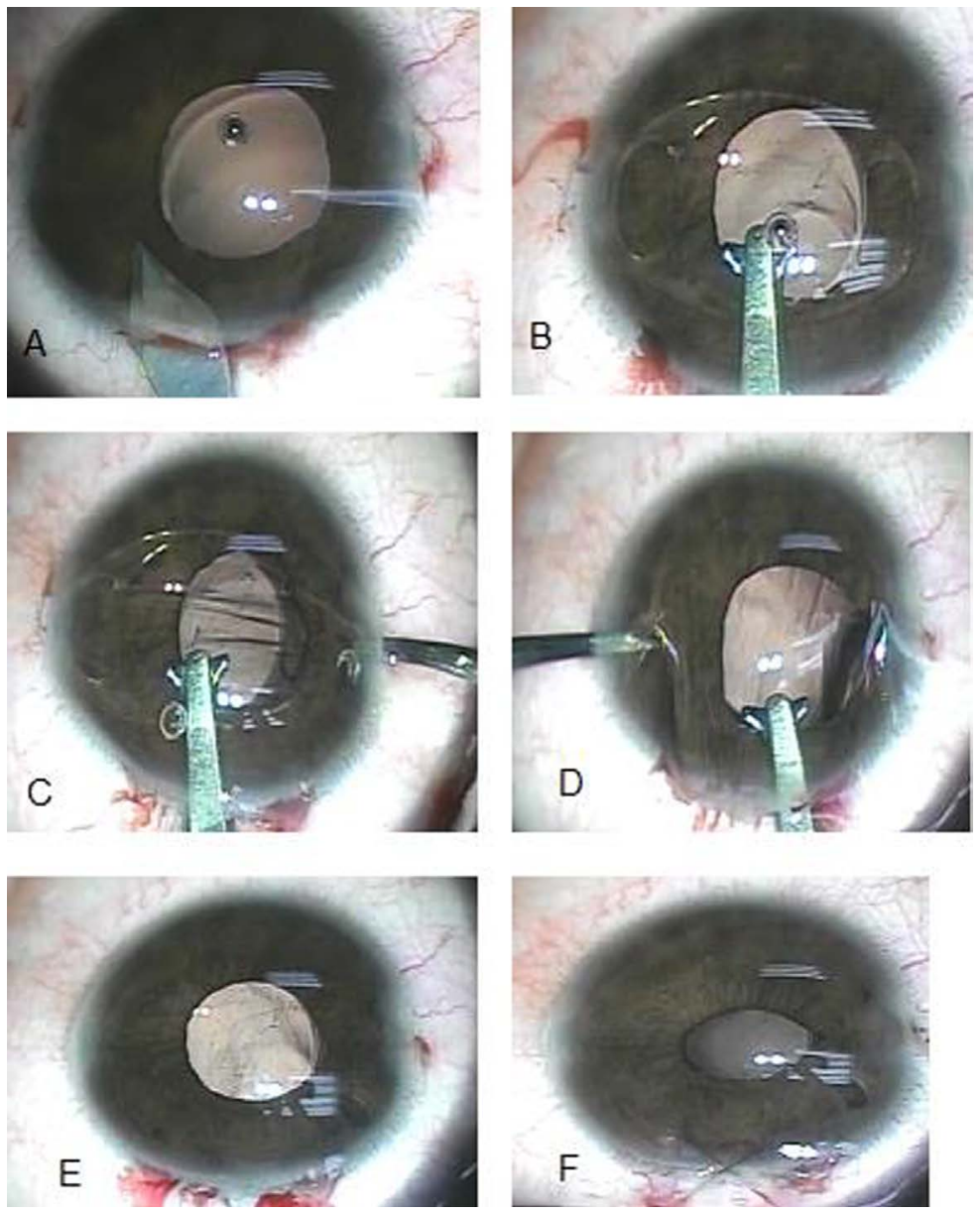

Figure 1. Intraoperative steps of retro-pupillary iris claw IOL implantation. (A) Superior $5.5 \mathrm{~mm}$ clear corneal incision was made. (B) IOL was inserted and held by artisan IOL forceps. (C) Enclavation of the iris into the right hand side claw. (D) Enclavation of the iris into the left hand side claw. (E)-(F) 10-0 nylon sutures were used to close the wound. 
had been kept outside the corneal incision. The tip of the leading haptic was then grasped with $25 \mathrm{G}$ end gripping micro rhexis forceps, pulled through the sclerotomy, and externalized. After the trailing haptic was introduced into the anterior chamber, the haptic tip was grasped with a 25 -gauge forceps, pulled through the second sclerotomy, and externalized on opposite side. The tip of the haptic was embedded into the limbal-parallel scleral tunnel, after which the IOL is positioned and centered. A single 8-0 nylon suture was used to fixate the haptic to the scleral bed to prevent it from shifting immediately after surgery and the sclerotomy was closed with 8-0 polyglactin 910 (Vicryl 8-0) (Figure 2).
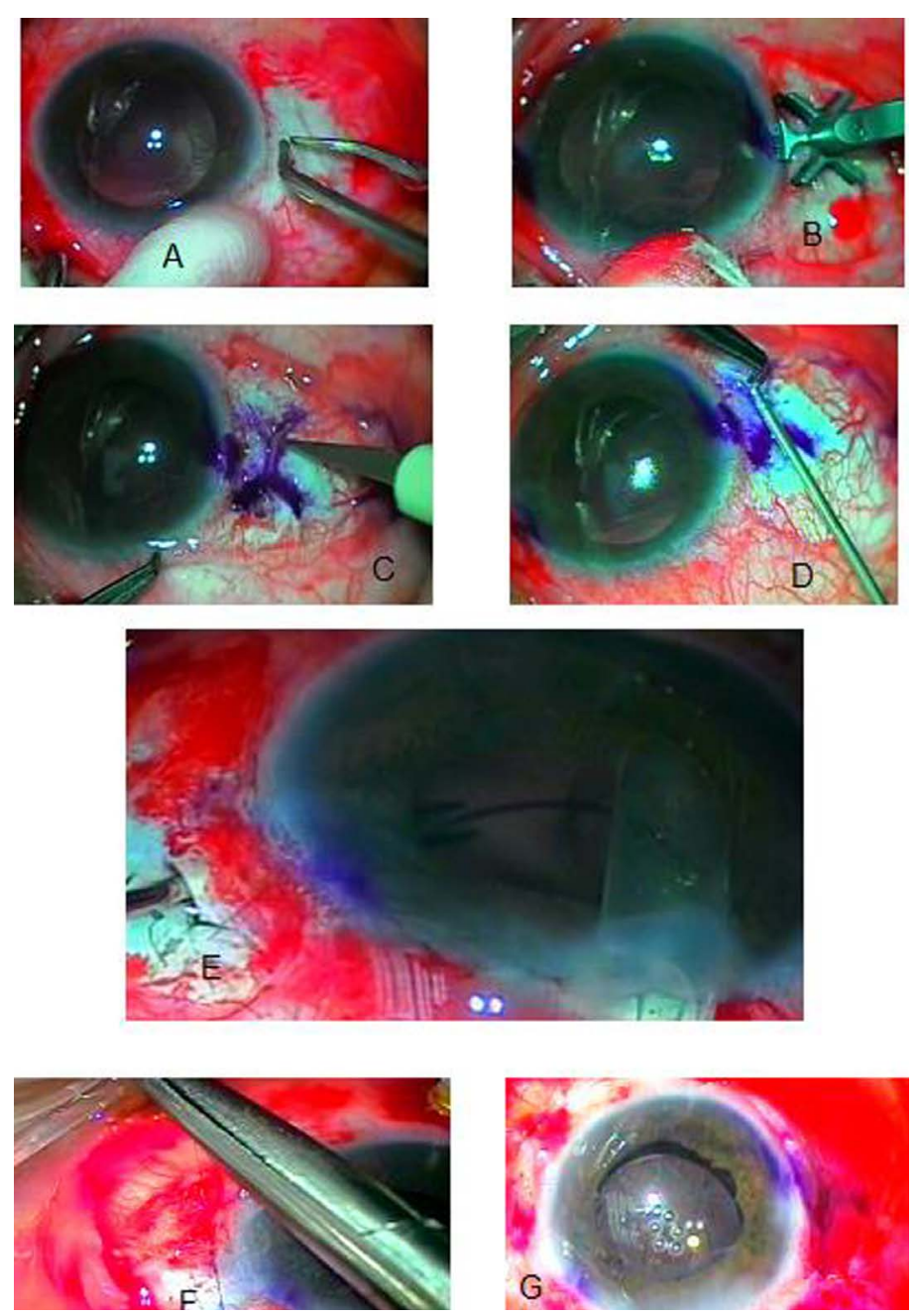

Figure 2. Intraoperative steps of Y-shaped intra-scleral fixated IOL implantation. (A) A $5.0 \mathrm{~mm}$ conjunctival peritomy is done with cauterization of sclera. (B) A reference marker and $\mathrm{Y}$ marker are used for marking. (C) Y-shaped incisions are made $1.5 \mathrm{~mm}$ from the limbus. (D) A scleral tunnel is made parallel to the limbus at the branching point of the Y-shaped incision. (E) The tip of the leading haptic is grasped with a 25-gauge IOL haptic gripping forceps, pulled through the sclerotomy, and externalized on the left side. (F) A single 8-0 nylon suture is used to fixate the haptic to the scleral bed. (G) Centrally positioned IOL with closure of conjunctiva. 

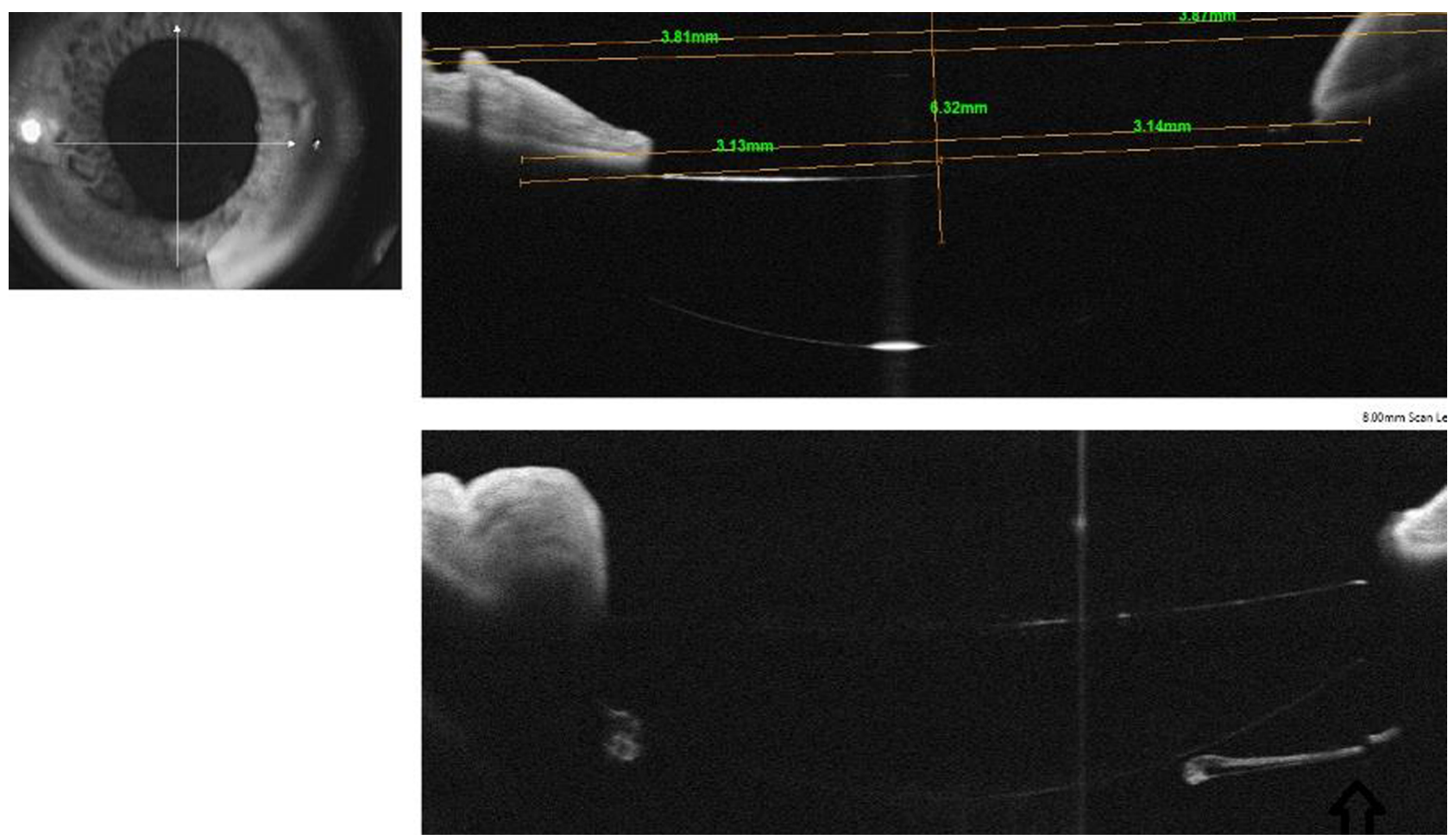

Figure 3. Anterior segment OCT showing centrally positioned retropupillary IOL with no IOL tilt. 
were tested for normality using Kolmogorov-Smirnov test, assuming normality at $\mathrm{P}>0.05$, the accepted level of significance in this work was stated at $0.05(\mathrm{P}<$ 0.05 was considered significant).

\section{Results}

This study was carried out on 100 eyes of 96 patients that were randomly distributed between 2 groups (A and B) according to their order of presentation. 50 eyes involved in each group. Three patients had bilateral implantation of retropupillary iris claw lens while one patient had bilateral implantation of Y-shaped intra-scleral fixated IOL. In group A, the age ranged from 18 to 62 years with a mean age of $43.8 \pm 15.2$ years, while in group $B$, the age ranged from 18 to 68 years with a mean age of $44.5 \pm 15.7$ years with no statistically significant difference between both groups ( $\mathrm{p}>0.05)$. Also both groups were matched in sex as 27 (57.4\%) of group A were males and 20 (42.6\%) females, while in group B, there were $32(65.3 \%)$ male and $17(34.7 \%)$ females with no statistically significant difference between both groups ( $p>0.05)$ (Table 1).

Fifty-three (53\%) eyes were Aphakic after complicated cataract surgery with inadequate capsular support for primary IOL implantation, (14\%) were aphakic after traumatic and congenital luxation of crystalline lens, twenty-one eyes (21\%) were Aphakic after globe rupture with sever posterior capsular tear, six eyes (6\%) were Aphakic after irrigation/aspiration for congenital cataract, 3 eyes (3\%) were aphakic after late dislocation of posterior chamber IOL, two of them had pre-existing pseudo exfoliation syndrome and three eyes (3\%) had a history of PPV with lensectomy for retinal detachment repair, removal of intra ocular foreign body (IOFB) and management of non-resorbing vitreous haemorrhage respectively, With no statistical significant difference among both groups regarding the cause (Figure 4).

The mean preoperative BCVA for group A was $0.21 \pm 0.13$ while the mean preoperative BCVA for group B was $0.19 \pm 0.12$. There was no significant statistical difference ( $p>0.05$ ). The IOP in group A, ranged from 10 to $21 \mathrm{~mm} \mathrm{Hg}$ in 41 cases; however 9 cases presented with IOP more than $21 \mathrm{~mm} \mathrm{Hg}$ with mean level of IOP $18.2 \pm 9.52 \mathrm{~mm} \mathrm{Hg}$, while in group B, the IOP ranged from 11 to 21 $\mathrm{mm} \mathrm{Hg}$ in 43 cases; however 7 cases presented with IOP more than $21 \mathrm{~mm} \mathrm{Hg}$

Table 1. Demographic data of the studied population.

\begin{tabular}{|c|c|c|c|c|c|c|}
\hline \multirow{3}{*}{$\begin{array}{c}\text { Variables } \\
\text { Sex }\end{array}$} & \multicolumn{4}{|c|}{ Frequency } & \multirow{3}{*}{$\mathrm{x}^{2} \backslash \mathrm{t}$-test ${ }^{*}$} & \multirow{3}{*}{$\mathrm{P}$-value } \\
\hline & \multicolumn{2}{|c|}{ Group $A=47$} & \multicolumn{2}{|c|}{ Group B $=49$} & & \\
\hline & $\mathrm{N}$ & $\%$ & $\mathrm{~N}$ & $\%$ & & \\
\hline Male & 27 & 57.4 & 32 & 65.3 & & 0.43 \\
\hline Female & 20 & 42.6 & 17 & 34.7 & 0.626 & NS \\
\hline Age/years & \multicolumn{2}{|c|}{$18-62$} & \multicolumn{2}{|c|}{$18-68$} & & 0.83 \\
\hline Mean \pm SD & \multicolumn{2}{|c|}{$43.8 \pm 15.2$} & \multicolumn{2}{|c|}{$44.5 \pm 15.7$} & $0.22^{\star}$ & NS \\
\hline
\end{tabular}

(S) P-value $<0.05$ is significant; (NS) p-value $>0.05$ is not significant. 


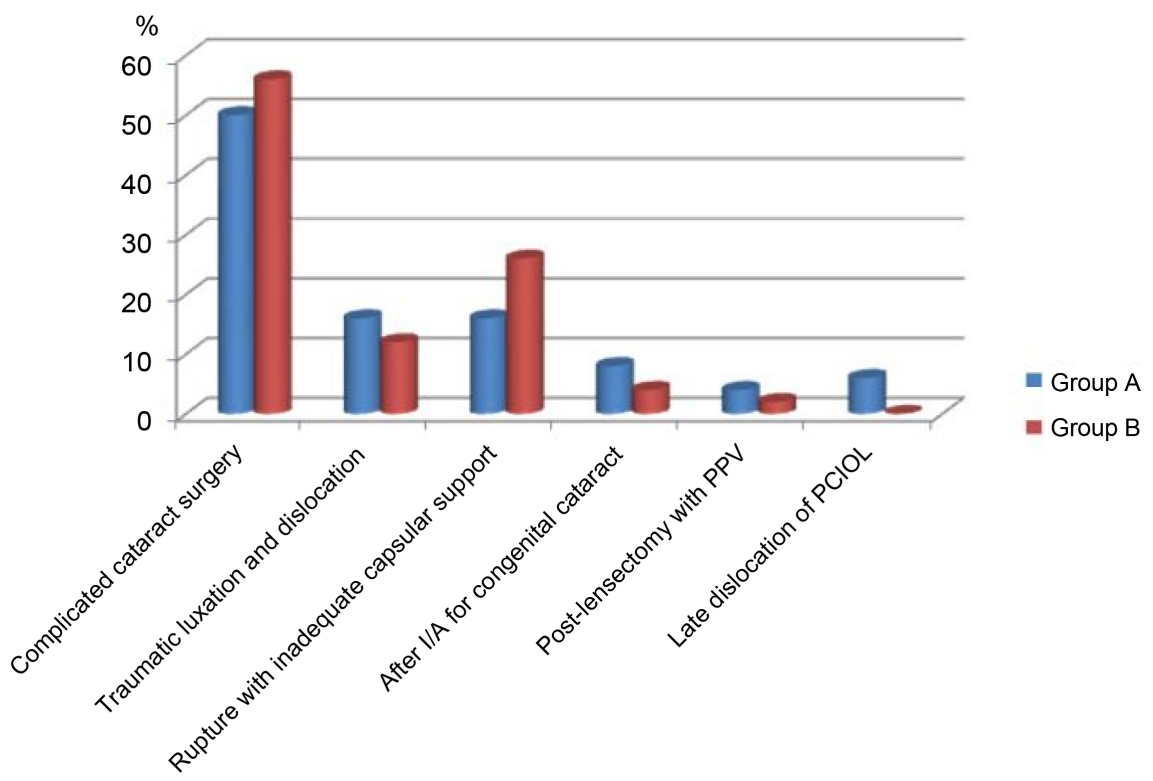

Figure 4. Bar chart showing percentage of the etiological classification of aphakia inside each group.

and the mean level was $17.5 \pm 7.7 \mathrm{~mm} \mathrm{Hg}$. Preoperative IOP more than $21 \mathrm{~mm}$ $\mathrm{Hg}$ in both groups was due to pupillary block glaucoma (anterior dislocation of crystalline lens or vitreous prolapse) and previous vitreo-retinal surgery (Table 2).

In group $\mathrm{A}$, the surgical duration ranged from 14 to $35 \mathrm{~min}$ with a mean of $21 \pm$ $5.3 \mathrm{~min}$, in group B, ranged from 39 to $64 \mathrm{~min}$ with a mean of $53.4 \pm 6.9 \mathrm{~min}$, with high statistically significant difference, (p-value $<0.001$ ) (Figure 5).

As regard intraoperative difficulties: Small intraoperative hyphema (less than $1 / 3$ of anterior chamber (AC) occurred in 6 eyes (12\%) in group A and 3 eyes (6\%) in group B. One of the two enclavation sites of the IOL loosened during intraoperative manipulations and the IOL luxated partially into the vitreous had been occurred in three cases in group $\mathrm{A}$, this can be prevented by intraoperative engagement of adequate iris tissue. ciliary bleeding occurred in $0(0 \%)$ in group A and 6 eyes (12\%) in group B. in group B, Mild kink of the haptics occurred in 6 cases (12\%) while Haptic break occurred in 2 cases (4\%). Anterior vitrectomy was done in $22(44 \%)$ cases in group A, 27 (54\%) cases of group B. intraoperative Hypotony occurred in 7 cases (14\%) in group A and 8 cases (16\%) in group B with no statistically significant difference between both groups $(\mathrm{p}>0.05)$ as regard intraoperative complications except for incidence of haptic kink in group $B$ which had statistically significant difference $(\mathrm{p}<0.05)$ (Table 3$)$.

Six months postoperative, the BCVA ranged from 0.08 to 0.6 in group A with mean of $0.34 \pm 0.15$. It ranged from 0.03 to 0.6 in group $B$ with mean of $0.31 \pm$ 0.16 with no statistically significant difference between both groups $(p>0.05)$ (Figure 6 \& Table 4).

The mean difference between the preoperative BCVA and 6 month follow up BCVA was 0.13 improvements in group A which was highly statistically significant 


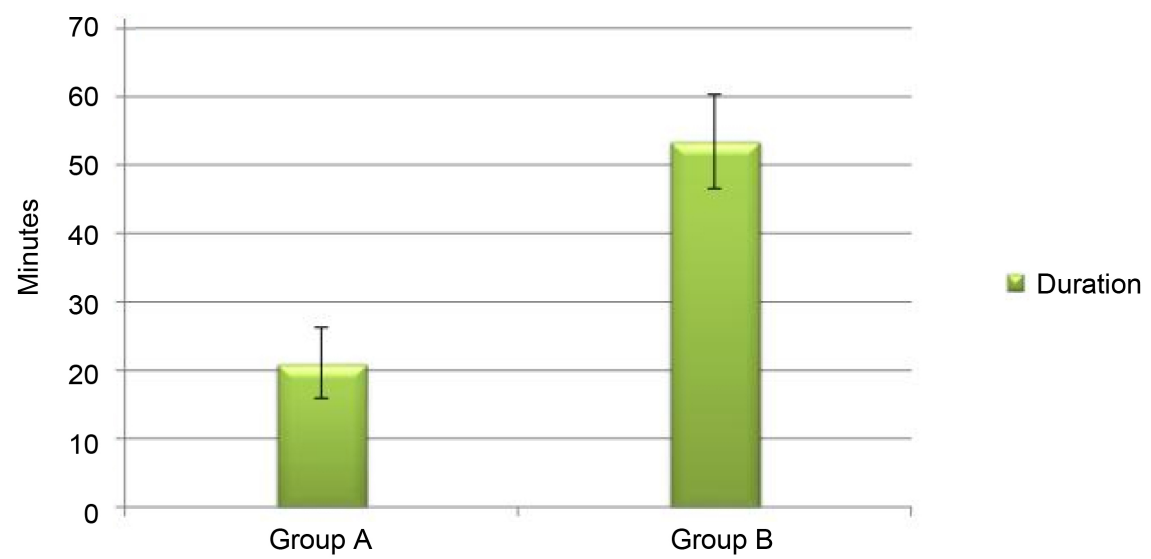

Figure 5. Bar chart showing surgical duration in minutes (Mean \pm SD) among both studied groups.

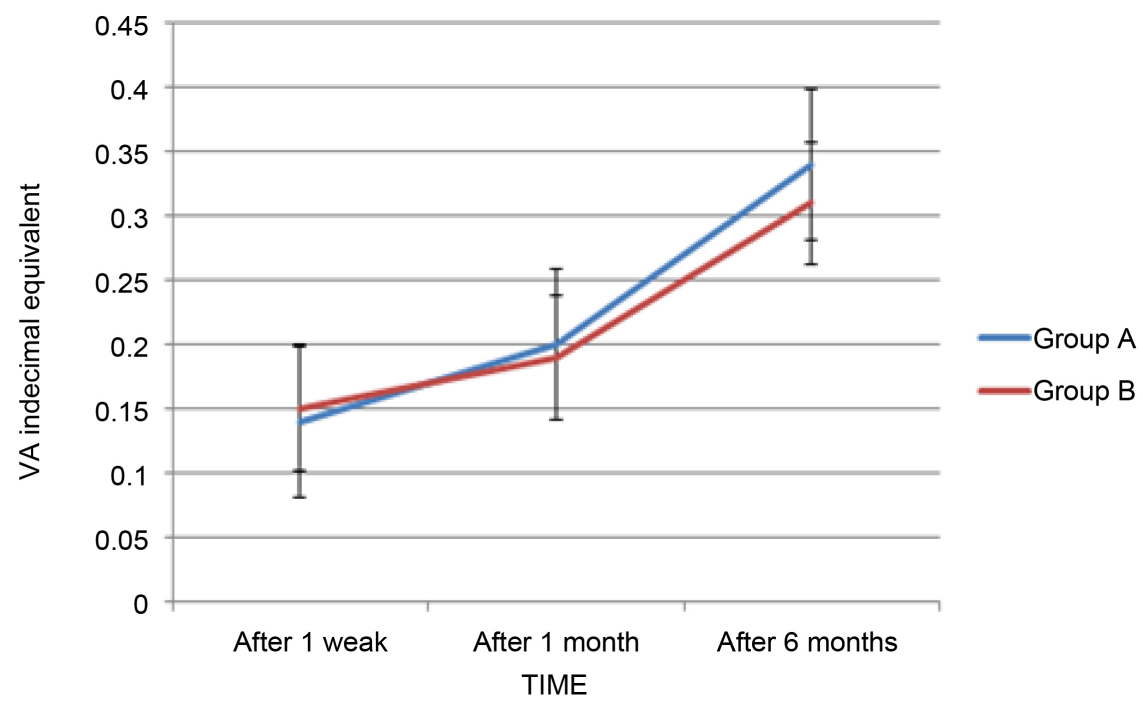

Figure 6. Line chart showing the mean post-operative BCVA among both studied groups.

Table 2. Pre-operative data among both studied groups.

\begin{tabular}{ccccc}
\hline \multirow{2}{*}{ Pre-operative data } & \multicolumn{2}{c}{ Frequency } & \multirow{2}{*}{ t-test $^{*}$} & P-value \\
\cline { 2 - 3 } & Group A $=\mathbf{4 7}$ & Group B $=\mathbf{4 9}$ & & \\
\hline BCVA & & & & \\
Mean \pm SD & $0.21 \pm 0.13$ & $0.19 \pm 0.12$ & 0.66 & 0.518 \\
Range & $0.01-0.6$ & $0.01-0.5$ & & NS \\
IOP & & & & \\
Mean \pm SD & $18.2 \pm 9.52$ & $17.5 \pm 7.7$ & 0.397 & 0.692 \\
Range & $10-50$ & $10-48$ & & NS \\
\hline
\end{tabular}

(S) P-value $<0.05$ is significant. (NS) P-value $>0.05$ is not significant.

results $(\mathrm{p}<0.001)$. Also the mean difference between the preoperative BCVA and 6 month follow up BCVA was 0.12 in group B which was statistically highly significant $(\mathrm{p}<0.001)$ (Figure 7$)$.

Six months postoperative, the IOP in group A ranged from 10 to 34 with a 
Table 3. Intraoperative data.

\begin{tabular}{ccccc}
\hline Intraoperative data & $\begin{array}{c}\text { Group A } \\
(\mathrm{n}=50 \text { eyes }) \\
\mathrm{N}(\%)\end{array}$ & $\begin{array}{c}\text { Group B } \\
(\mathrm{n}=50 \text { eyes }) \\
\mathrm{N}(\%)\end{array}$ & $\mathrm{x}^{2}$ & P value \\
\hline Intraoperative hypotony & $7(14 \%)$ & $8(16 \%)$ & 0.231 & 0.623 \\
Anterior vitrectomy & $22(44 \%)$ & $27(54 \%)$ & 0.078 & 0.779 \\
Small intraoperative hyphema & $6(12 \%)$ & $3(6 \%)$ & Fisher & 0.295 \\
Minor ciliary bleeding & $0(0 \%)$ & $4(8 \%)$ & Fisher & $0.05 \mathrm{NS}$ \\
Major ciliary bleeding & $0(0 \%)$ & $2(4 \%)$ & Fisher & $0.153 \mathrm{NS}$ \\
Haptic Break & $0(0 \%)$ & $2(4 \%)$ & Fisher & $0.153 \mathrm{NS}$ \\
Haptic Kink & $0(0 \%)$ & $6(12 \%)$ & Fisher & $0.03 \mathrm{~S}$ \\
Intra-operative Partial & $3(6 \%)$ & $0(0 \%)$ & Fisher & $0.153 \mathrm{NS}$ \\
subluxation of IOL & $0(0 \%)$ & $3(6 \%)$ & Fisher & $0.08 \mathrm{NS}$ \\
\hline $\begin{array}{c}\text { Decentration of the IOL } \\
\text { Mel }\end{array}$ & & &
\end{tabular}

(S) P-value $<0.05$ is significant. (NS) P-value $>0.05$ is not significant.

Table 4. Post-operative BCVA among both studied groups.

\begin{tabular}{ccccc}
\hline \multirow{2}{*}{ BCVA } & \multicolumn{2}{c}{ Frequency } & \multirow{2}{*}{ t-test ${ }^{*}$} & P-value \\
\cline { 2 - 3 } & Group A $=47$ & Group B $=49$ & & \\
\hline After 1 weak & $0.14 \pm 0.06$ & $0.15 \pm 0.07$ & 1.16 & 0.25 NS \\
Mean SD & $0.08-0.3$ & $0.05-0.3$ & & \\
Range & & & & \\
After 1 month & $0.2 \pm 0.11$ & $0.19 \pm 0.103$ & 0.45 & $0.652 \mathrm{NS}$ \\
Mean \pm SD & $0.08-0.5$ & $0.05-0.5$ & & \\
Range & & & & \\
After 6 month & $0.34 \pm 0.15$ & $0.31 \pm 0.16$ & 0.741 & $0.47 \mathrm{NS}$ \\
Mean \pm SD & $0.08-0.6$ & $0.03-0.6$ & & \\
Range & & & & \\
\hline
\end{tabular}

(NS) P-value $>0.05$ is not significant.

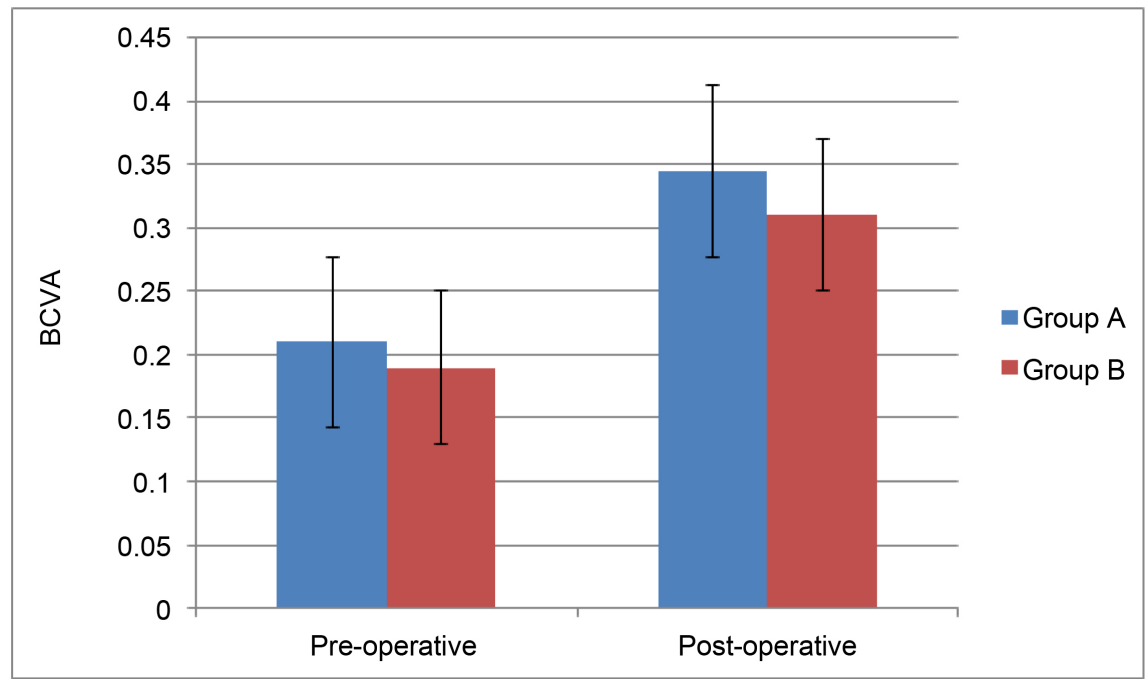

Figure 7. Bar chart showing preoperative and post-operative BCVA among both studied groups. 
mean of $13.4 \pm 4.02 \mathrm{~mm} \mathrm{Hg}$; in group B it ranged from 10 to 32 with a mean of $14.4 \pm 4.6 \mathrm{~mm} \mathrm{Hg}$, With no statistically significant difference between both groups in all post-operative IOP levels ( $p>0.05)$ (Table 5).

Corneal oedema occurred in $9(18 \%)$ cases in group A and $6(12 \%)$ cases in group B, all cases respond to topical hypertonic agents. Anterior chamber reaction occurred in 13 (26\%) cases in group A and $5(10 \%)$ cases in group B with significant difference between groups ( $\mathrm{p}$ value $<0.05$ ). Pupillary distortion: 10 (20\%) cases in group A and $6(12 \%)$ cases in group B showed pupillary distortion with variable degree. Vitreous haemorrhage occurred in $1(2 \%)$ case in group A and in $6(12 \%)$ cases in group B, mild to moderate vitreous haemorrhage were detected that were followed up till resolved. IOL decentration was observed in $1(2 \%)$ in group A and 7 (14\%) cases in group B, with statistically significant difference, $(\mathrm{p}$ value $=0.03$ ). However IOL decentration was mild (between $0.5-0.8 \mathrm{~mm}$ ) in the majority of cases and has no effect on the final visual outcome. IOL tilt occurred in $0(0 \%)$ cases in group A and $5(10 \%)$ cases in group B with statistically significant difference, $(\mathrm{p}$ value $=0.02)$. CME was detected in cases with unexplained visual deterioration after initial improvement during follow up in $2(4 \%)$ cases in group A and $5(10 \%)$ cases of group B with no statistical significant difference (Table 6).

Table 5. Post-operative IOP among both studied groups.

\begin{tabular}{ccccc}
\hline \multirow{2}{*}{ IOP } & \multicolumn{2}{c}{ Groups of patients } & \multirow{2}{*}{ t-test } & P-value \\
\cline { 2 - 3 } & Group A $=47$ & Group B $=49$ & & \\
\hline After 1 weak & $13.4 \pm 4.86$ & $14.3 \pm 5.87$ & 0.902 & $0.365 \mathrm{NS}$ \\
Mean SD & $10-38$ & $10-38$ & & \\
Range & $12.96 \pm 2.7$ & $13.7 \pm 3.91$ & \multirow{2}{*}{1.11} & 0.27 NS \\
After 1 month & $10-27$ & $10-30$ & & \\
Mean \pm SD & & & & \\
Range & $13.4 \pm 4.02$ & $14.4 \pm 4.6$ & 1.17 & 0.24 NS \\
After 6 month & $10-34$ & $10-32$ & & \\
Mean \pm SD & & & \\
Range & &
\end{tabular}

(NS) P-value $>0.05$ is not significant.

Table 6. Post-operative complications among both studied groups.

\begin{tabular}{cccccc}
\hline & $\begin{array}{c}\text { Group A } \\
(50 \text { eyes })\end{array}$ & $\begin{array}{c}\text { Group B } \\
(50 \text { eyes })\end{array}$ & \multirow{2}{*}{$\mathrm{x}^{2}$} & P value \\
\cline { 2 - 3 } & $\mathrm{N}(\%)$ & $\mathrm{N}(\%)$ & & \\
\hline Corneal edema & $9(18)$ & $6(12)$ & & 0.701 & $0.402 \mathrm{NS}$ \\
Anterior chamber location & $13(26 \%)$ & $5(10 \%)$ & & 4.34 & $0.04 \mathrm{~S}$ \\
Pupillary distortion & $10(20 \%)$ & $6(12 \%)$ & & 1.19 & $0.275 \mathrm{NS}$ \\
Vitreous haemorrhage & $1(2 \%)$ & $6(12 \%)$ & & Fisher & $0.05 \mathrm{NS}$ \\
IOL decentration $(0.5-1.00) \mathrm{mm}$ & $1(2 \%)$ & $7(14 \%)$ & & Fisher & $0.03 \mathrm{~S}$ \\
CME & $2(4 \%)$ & $5(10 \%)$ & & Fisher & $0.24 \mathrm{NS}$ \\
IOL tilt & 0 & $5(10 \%)$ & Fisher & $0.02 \mathrm{~S}$ \\
Haptic extrusion & 0 & $2(4 \%)$ & & Fisher & $0.08 \mathrm{NS}$ \\
\hline
\end{tabular}

(S) P-value $<0.05$ is significant. (NS) P-value $>0.05$ is not significant. 
There was a statistical significant difference among both groups as regard mean difference in endothelial cell count pre- and post-operative which was higher among group B with mean of $64.5 \pm 47.6$ and mean of $28.7 \pm 26.6$ among group A. Also percent of change was higher among group B with mean of $2.8 \pm$ 1.9 , while among patients of group A the mean was $1.28 \pm 1.2$ with statistical significant difference among both groups (Table 7).

\section{Discussion}

The management of Aphakic eyes without sufficient capsular support is still difficult. Different treatment lines had been suggested, for example correction with contact lenses or glasses or secondary IOL implantation. As regard secondary IOL implantation, the technique which offers the lowest complication rate combined with the best possible visual result ought to be chosen [5].

Artisan/Verisyse aphakia IOLs (OPHTEC B. V./Advanced Medical Optics, Inc.) are the new generation of iris-fixated IOLs that are attached to the mid periphery of the iris. With the new design of the Artisan aphakic iris claw IOL, direct contact between the IOL and the iris had been avoided (with the exception of the clamping sides), so the possibility of iris trauma and postoperative inflammation also had been decreased [6].

Trans-scleral fixation of posterior chamber isn't without complications e.g. endophthalmitis related to disintegration of the fixation sutures through the conjunctiva, IOL dislocation related to suture breakage, vitreous hemorrhage, and IOL tilt. To abolish such suture-related problems, Gabor et al. (2007) described a method for intra scleral fixation of both haptics in the ciliary sulcus using a parallel scleral tunnel, with a 24-gauge needle utilized to make a straight sclerotomy [4].

In the present work, the results of retropupillary iris claw fixated IOL technique (group A) was compared to the Y-shaped intra scleral fixated IOL

Table 7. Pre- and post-operative endothelial cell count among both studied groups.

\begin{tabular}{|c|c|c|c|c|}
\hline \multirow{2}{*}{ Endothelial cell count } & \multicolumn{2}{|c|}{ Groups of patients } & \multirow{2}{*}{ t-test ${ }^{*}$} & \multirow{2}{*}{$\mathrm{P}$-value } \\
\hline & Group $\mathrm{A}=47$ & Group B = 49 & & \\
\hline \multicolumn{5}{|l|}{ Pre-operative } \\
\hline Mean SD & $2157.7 \pm 361.8$ & $2193.7 \pm 384.8$ & 0.342 & $0.734 \mathrm{NS}$ \\
\hline Range & $1560-2874$ & $1514-2519$ & & \\
\hline \multicolumn{5}{|l|}{ Post-operative } \\
\hline Mean \pm SD & $2128.9 \pm 356.5$ & $2129.6 \pm 360.9$ & 0.01 & $0.995 \mathrm{NS}$ \\
\hline Range & $1555-2845$ & $1440-2803$ & & \\
\hline \multicolumn{5}{|l|}{ Level of difference } \\
\hline Mean \pm SD & $28.7 \pm 26.6$ & $64.5 \pm 47.6$ & 3.29 & $0.002 \mathrm{~S}$ \\
\hline Range & $2-108$ & $11-200$ & & \\
\hline \multicolumn{5}{|l|}{ Percent of change } \\
\hline Mean \pm SD & $1.28 \pm 1.2$ & $2.8 \pm 1.9$ & 3.25 & $0.001 \mathrm{~S}$ \\
\hline Range & $0.1-5$ & $0.5-8.3$ & & \\
\hline
\end{tabular}

(S) P-value $<0.05$ is significant. (NS) P-value $>0.05$ is not significant. 
technique (group B); evaluated in 100 patients that were randomly and equally distributed between both groups and followed for at least 6 months.

In the present study, (53\%) of eyes were Aphakic after complicated cataract surgery with inadequate capsular support for primary IOL implantation, (14\%) were aphakic after traumatic and congenital luxation of crystalline lens, (21\%) were Aphakic after globe rupture with sever posterior capsular tear, (6\%) were Aphakic after irrigation/aspiration for congenital cataract, 3 eyes (3\%) were aphakic after late dislocation of posterior chamber IOL, two of them had pre-existing pseudo exfoliation syndrome and three eyes (3\%) had a history of PPV with lensectomy for retinal detachment repair, removal of intra ocular foreign body (IOFB) and management of non-resorbing vitreous haemorrhage.

In the present study, the surgical time in (Group A) ranged from 14 to $35 \mathrm{~min}$ with a mean of $21 \pm 5.3 \mathrm{~min}$, while in group $\mathrm{B}$, ranged from 39 to 64 min with a mean of $53.4 \pm 6.9 \mathrm{~min}$, with high statistically significant difference, (p-value $<$ $0.001)$.

As regard intraoperative hyphema; in group A, (12\%) of eyes were reported with mild hyphema, while in group B, $(6 \%)$ of eyes were reported with mild to moderate hyphema. High incidence of hyphema in group A attributed to intraoperative iris manipulation, all of these cases resolved spontaneously with no long lasting effects.

In the present study, in group B, intraoperative haptic breakage was reported in (4\%) of eyes that necessitated an IOL replacement with another IOL, While haptic deformation was reported in (12\%) of cases. This problem can be avoided by using a forceps with a smooth tip not a ridged tip to hold the haptic during externalization from its tip; this is encouraged by the hand shake technique [3].

In the present study, in group A, One of the two enclavation sites of three (6\%) artisan IOLs loosened during the intraoperative manipulations and the IOL partially dropped into the vitreous. The lens had been elevated by a 23 G MVR blade (DORC, Netherlands) introduced through a sclerotomy $3.5 \mathrm{~mm}$ behind the limbus, grasped with the artisan lens forceps and re-enclavated again. This problem can be avoided by intra-operative enclavation of adequate iris tissue in the claw. As regarding intraoperative hypotony, (14\%) of eyes in group A and (16\%) in group B showed intraocular hypotony. The hypotony occurred in cases where extensive vitrectomy was made especially in young age patients, and this was solved by anterior chamber maintainer (ACM) in Y-shaped intra-scleral fixated IOL group while in retro-pupillary IOL group was treated by repeated injection of ophthalmic viscosurgical devices (OVD) e.g. Healon GV.

BCVA significantly deteriorated 1 day after surgery in both groups compared with preoperative BCVA. This apparently happened due to intraoperative manipulations and early postoperative complications including (corneal oedema, hyphema, anterior chamber reaction and ciliary body hemorrhage). However eyes managed with implantation of retropupillary iris claw IOL showed earlier visual recovery than in intra scleral fixated group. 
In group A, the final BCVA was better than preoperatively in (78\%) of eyes, unchanged in $(16 \%)$, and was worse in $(6 \%)$. On average, the mean difference between the preoperative BCVA and 6 month follow up BCVA was 0.13 improvements in group A which was highly statistically significant results ( $\mathrm{p}<$ $0.001)$. In group B, the final BCVA was better than preoperatively in (88\%) eyes, unchanged in $(8 \%)$, and was worse in $(4 \%)$ of eyes. On average, the mean difference between the preoperative BCVA and 6 month follow up BCVA was 0.12 in group B which was statistically high significant $(\mathrm{p}<0.001)$.

Six months postoperative, the BCVA ranged from 0.08 to 0.6 in group A with mean of $0.34 \pm 0.15$. It ranged from 0.03 to 0.6 in group $B$ with mean of $0.31 \pm$ 0.16 with no statistically significant difference between both groups ( $p>0.05)$.

Accurate measurement of IOP in first postoperative day was difficult in both groups, however in both groups high IOP in the early postoperative period was probably secondary to postoperative inflammation, iris pigment release due to intraoperative manipulations, iris manipulations and residual viscoelastic substance in the anterior chamber. All cases received topical steroid, topical antiglaucoma (combination Brimonidine $0.2 \%$ plus Timolol 0.5) and systemic medications e.g. Cidamex $250 \mathrm{mg}$ tablet twice/day are given in the first 3 days.

After the first postoperative week the mean of IOP in group A was $13.4 \pm 4.86$ $\mathrm{mmHg}$, in group B it was $14.3 \pm 5.87 \mathrm{~mm} \mathrm{Hg}$. After the first postoperative month, the mean of IOP in group A was $12.96 \pm 2.7 \mathrm{~mm} \mathrm{Hg}$; in group B it was $13.7 \pm 3.91 \mathrm{~mm} \mathrm{Hg}$. At the last visit, the mean IOP in group A was $13.4 \pm 4.02$ $\mathrm{mmHg}$; in group B it was $14.4 \pm 4.6 \mathrm{~mm} \mathrm{Hg}$, with no statistically significant difference between both groups in all post-operative IOP levels ( $p>0.05)$. In our study, we found elevated IOP in seven cases, two cases in group A and five cases in group $\mathrm{B}$.

In group A, high postoperative IOP occurred in two patients (4\%), the first patient presented with IOP fluctuating between (28 - $34 \mathrm{mmhg}$ ) 6-days postoperative (mainly steroid induced) and was managed by systemic, topical anti glaucoma medications and discontinuation of topical steroids. The second patient presented with postoperative IOP more than $30 \mathrm{~mm} \mathrm{Hg}$ and poorly controlled with antiglaucoma medications with progressive structural damage to optic nerve and underwent glaucoma filtering surgery (GFS). Peripheral iridectomies hadn't been done in any case with retropupillary IOL and no cases of pupillary block had occurred. This could be clarified by the posterior vaulting of iris claw lens when placed in a reverse position on the back of the iris and the sufficient space between the lens optic and the back of the iris.

In group B, high postoperative IOP occurred in five patients(10\%), three of them were controlled by systemic, topical anti glaucoma medications, One patient presented with late increase in IOP (after 3 months postoperative) and controlled medically. The last patient had previous PPV and was preoperatively had a high IOP (fluctuating between 22 - 30), this patient presented with persistent uncontrolled elevation of postoperative IOP with progressive deterioration of visual functions and end by BCVA less than 0.03 after GFS. 
With respect to postoperative complications in this study. Mild anterior chamber reaction occurred in (26\%) of eyes with iris claw lens and (10\%) of eyes in scleral fixation group, with significant difference between groups. This can be explained by vigorous intraoperative iris manipulation in group A, all cases had been cleared with topical steroids.

Transient corneal oedema occurred in (18\%) of cases in group A and (12\%) of cases in group B, all cases respond to topical hypertonic agents. Postoperative pupillary distortion occurred in (20\%) of cases in group A and (12\%) of cases in group B. Pupil Ovalization in group A, which could be explained by too tight enclavation in midperipheral iris stroma (or asymmetric enclavation on both sides), tended to normalize over time. pupillary distortion in group B was due to preoperative irregular pupils. To prevent pupillary distortion, iris-claw IOL should be fixed in the mid-peripheral part of the iris and both the claws should be clamped symmetrically on the iris with equal volume of iris tissue.

In the present study, vitreous haemorrhage occurred in (2\%) of eyes in group A and (12\%) of eyes in group B, that were followed up till resolved. Haptic extrusion occurred in (4\%) of intra scleral fixated IOLs group (group B).

CME was detected in cases with unexplained visual deterioration during follow up in (4\%) of cases in group A and (10\%) cases of group B with no statistical significant difference. All cases were treated by topical treatment (steroidal and non-steroidal anti-inflammatory eye drops), oral cidamex with good improvement.

In this work, IOL decentration was observed in (2\%) of eyes in group A and $(14 \%)$ in group $B$, with statistically significant difference, ( $\mathrm{p}$ value $=0.03$ ). however all cases with minimal IOL decentration $(0.5-1 \mathrm{~mm})$ requiring no repositioning since the patient had good visual acuity and did not complain about halos. No cases of postoperative IOL dislocation were recorded in either group. IOL tilt is one of the components of malposition that can lead to astigmatism, change in optical higher-order aberrations, and loss of best-corrected visual acuity. In this work, IOL tilt was observed in (0\%) in group A and (10\%) in group B, with statistically significant difference, $(\mathrm{p}$ value $=0.02)$.

In the present study, it was a statistical significant difference among both groups as regard mean difference in endothelial cell count (pre- and post-operative) which was higher among group B with mean of $64.5 \pm 47.6$ and mean of $28.7 \pm 26.6$ among group A. Also percent of change was higher among group $B$ with mean of $2.8 \pm 1.9$, while among patients of group A the mean was $1.28 \pm 1.2$ with statistical significant difference among both groups. High incidence of endothelial cell loss in group B attributed to intraoperative manipulations with long surgical duration, No clinical signs of a corneal decompensation had been detected in any patients of both groups.

Hara, et al. (2011) reported that, the Mean surgical time of Verisyse implantation $(20.0 \pm 8.9 \mathrm{~min})$ was significantly shorter than scleral fixation group $(49.7 \pm$ $18.9 \mathrm{~min}$ ), which was consistent with results of this study [7]. 
As regard the intraoperative data in Y-shaped intra-scleral fixation (group B): Kumar DA et al. (2013) [8] found in their study intraoperative hyphema in $(0.4 \%)$ of cases which was inconsistent with this work, however Agarwal et al. (2011) [9] as well as Narang and Narang (2013) [10] found results similar to our study. Kumar DA et al. (2013) [8] found in their study intraoperative haptic breakage in $(0.4 \%)$ of cases, and deformed haptics in $(0.9 \%)$ which was inconsistent with this work. McKee et al. (2014) [11] reported a self-resolving hypotony occurred in (22\%) of cases which was consistent with our results. In retro-pupillary iris claw IOL group (group A), incidence of intraoperative One haptic dis-enclavation with partial IOL subluxation was comparable with results reported by Brandner M et al. (2015) [12].

In series by KS Lett and PR Chaudhuri (2011) [13], (66.6\%) of patients achieved a final visual acuity better than the pre-operative best recorded visual acuity. A total of 12 eyes (30.8\%) achieved a final acuity equal to that measured pre-operatively, and only 1 eye (2.6\%) ended up with a poorer final acuity, Rao and Sasidharan (2013) [14] reported that, Majority i.e. 80\% of patients, had visual acuity of 20/40 or better at the end of follow up period. These results were consistent with our results in group A as regard postoperative BCVA.

In series by Narang and Narang (2013) [10], about $84 \%$ of the eyes had a gain of one or more lines, $12 \%$ had no gain, and $4 \%$ ended up with a poorer final acuity. This was consistent with our results in group B.

Six months postoperative, there was no statistically significant difference in BCVA between both groups in all post-operative period ( $p>0.05$ ), this was consistent with Menezo et al. (1996) [15] and recently Maher Saleh et al. (2013) [16] who reported a series of 26 eyes in 23 patients who were divided into 2 groups (Group 1: intra-scleral IOLs [Acrysof MN60 AC]; Group 2: retropupillary iris-claw IOLs [Verisyse]). The main outcome was the final visual acuity. The final CDVA was not different between groups ( $p>0.05$ ).

Johannes Gonnermann et al. (2012) [17] reported, (4.3\%) of cases with IOP elevation (more than $20 \mathrm{~mm} \mathrm{Hg}$ ) with posterior iris claw IOL which was similar to our results in (group A). Compared to our results in group B; Scharioth GB et al. (2010) [18] reported on results of intra scleral fixated IOL; there was no post-operative secondary glaucoma in any of the eyes. Also Narang and Narang (2013) [10] reported raised IOP (>20 mm Hg) in (4\%) of patients with glued intra scleral fixated IOL.

With regard to complications in this work. Hazar et al. (2013) [19] reported that, $(20.8 \%)$ of cases after retropupillary iris fixed IOL and (9.6\%) of cases after scleral fixed IOL implantation suffered from anterior chamber reaction, this was consistent with our work. Shanida H. S. et al. (2018) [20] reported, (36\%) of cases with iritis in the first 2 weeks of surgery with posterior iris claw IOL which was higher than our results in (group A). In contrary to our results; Kumar DA et al. (2013) [8] reported grade 2 anterior chamber reaction in (2.4\%) of cases with intra-scleral fixated IOL. 
Compared to our results about postoperative corneal oedema; Hazar et al. (2013) [19], reported lower rate of corneal oedema, (4.1\%) in retropupillary iris claw group than in scleral fixation group (9.6\%). Also In contrary to our results; Kumar DA et al. (2013) [8] reported lower rate of corneal edema (5.7\%) in intra-scleral fixated group.

Gonnermann et al. (2012) [17] found persistent pupil ovalization in (13.9\%) of cases in retro pupillary iris claw group which was Comparable with this study. In contrary to our results Hazar et al. (2013) [19] reported 0 (0\%) cases with pupillary distortion in retropupillary iris claw group.. However in scleral fixation group, our results were comparable with him as pupillary distortion occurred in $4(12.9 \%)$ cases.

In the present study, vitreous hemorrhage occurred in (2\%) of eyes in group A and $(12 \%)$ of eyes in group B, our results were comparable with Hazar et al. (2013) [19] who reported (4.1\%) cases of hemorrhage in retropupillary iris claw group while they reported (6.4\%) eyes of vitreous hemorrhage in scleral fixation cases. In contrary to our results in group B, Toshihiko Ohta et al. (2014) [21] reported $(6 \%)$ of cases of vitreous hemorrhage with IOL intra-scleral Fixation, also Yamane S et al. (2014) [22] reported (5\%) cases with vitreous hemorrhage in intra-scleral Fixation group.

Haptic extrusion occurred in (4\%) of intra scleral fixated IOLs group (group B), however Kumar DA et al. (2013) [8] reported haptic extrusion in (1.9\%) of cases with intra-scleral fixated IOL.

CME was detected in cases with unexplained visual deterioration during follow up in (4\%) of cases in group A and (10\%) of cases in group B with no statistical significant difference. In contrary to our study, Shuya Hara et al. (2011) [7] recorded no cases of CME in the Verisyse IOL group, while it was recorded in 1 (3.2\%) case in the scleral fixation group, however In a retrospective case series by Gonnermann et al. (2012) [17], CME was detected in retro pupillary iris claw group in (8.7\%) of cases, which was higher than our results. Kumar et al. (2013) [8] stated that CME occurred in $1.9 \%$ of eyes with foldable-glued IOL postoperatively. Likewise, cystoid macular edema was seen in $1.0 \%$ of eyes in results reported by Yamane S et al. (2014) [22]. These findings were inconsistent with our results in group $\mathrm{B}$.

In this work, IOL decentration was observed in (2\%) of eyes in group A and $(14 \%)$ in group $B$, with statistically significant difference, ( $p$ value $=0.03$ ). In contrary to this, Scharioth GB et al. (2010) [18] reported intraocular lens decentration occurred in $(3.6 \%)$ of cases in intra-scleral fixation cases. Also Kumar et al. (2013) [8] reported a lower rate of IOL decentration (3.3\%), with glued intra-scleral fixated PC IOL.

In the current study, No cases of IOL dislocation were recorded in either group. Our results were consistent with the results obtained by Hara et al. (2011) [7] and Rao et al. (2013) [14] who reported 0 (0\%) case of dislocation after retropupillary iris claw IOL implantation and only 1 (5.8\%) after scleral fixed IOL implantation. Yamane S et al. (2014) [22] reported no cases with post-operative 
IOL dislocation in intra-scleral Fixation group. As regard IOL tilt, it was a statistical significant difference among both groups, as it was higher among group $\mathrm{B}$.

In the present work, it was statistical significant differences among both groups as regard mean difference in endothelial cell count pre- and post-operative which was higher among group B. No clinical sign of a corneal decompensation was detected in any patients of both groups. Our results were comparable with Rijneveld et al. (1994) [23] who described the option of retropupillary fixation of an iris-claw IOL in adults in 1994. This technique has the advantage that the IOL is located behind the iris and therefore the negative influence on the corneal endothelium is less than with anterior chamber IOLs.

Yamane S et al. (2014) [22] reported that, The mean corneal endothelial cell density decreased from 2341 cells $/ \mathrm{mm}^{2}$ before surgery to 2313 cells $/ \mathrm{mm}^{2}, 2240$ cells $/ \mathrm{mm}^{2}, 2189$ cells $/ \mathrm{mm}^{2}$, and 2244 cells $/ \mathrm{mm}^{2}$ at $6,12,24$, and 36 months, respectively ( $\mathrm{p}<0.01, \mathrm{p}<0.01, \mathrm{p}<0.01$, and $\mathrm{p}=0.17$, respectively).

\section{Limitations in This Study}

Postoperative follow up was only 6 months in this work so further studies are required to evaluate the endothelial cell loss and postoperative IOL stability in a long-term follow-up after retropupillary Artisan-IOL and intra-scleral fixated IOL.

\section{Conclusion}

The results of our study indicated that both methods are satisfactory in correcting aphakia without sufficient capsular support as regards postoperative (BCVA); however the surgical technique of retro-pupillary iris claw lens is easier, shorter, with low intra- and postoperative complications and safer than those used for intra-scleral fixation of IOL. But for eyes which lack both iris and capsular support, a scleral fixation of a posterior chamber IOL remains the only option.

\section{Conflicts of Interest}

The authors declare no conflicts of interest regarding the publication of this paper.

\section{References}

[1] Acar, N., Kapran, Z., Altan, T., Kucuksumer, Y., Unver, Y.B., et al. (2010) Secondary Iris Claw Intraocular Lens Implantation for the Correction of Aphakia after Pars Plana Vitrectomy. Retina, 30, 131-139. https://doi.org/10.1097/IAE.0b013e3181b32eef

[2] Kirby, D.B. (1999) Procedures in Intra Capsular Cataract Extraction: A New Method. Transactions of the American Ophthalmological Society, 39, 150-157.

[3] Agarwal, A., Kumar, D.A., Jacob, S., et al. (2008) Fibrin Glue-Assisted Sutureless Posterior Chamber Intraocular Lens Implantation in Eyes with Deficient Posterior Capsules. Journal of Cataract \& Refractive Surgery, 34, 1433-1438. 
https://doi.org/10.1016/j.jcrs.2008.04.040

[4] Gabor, S.G. and Pavlidis, M.M. (2007) Sutureless Intra-Scleral Posterior Chamber Intraocular Lens Fixation. Journal of Cataract \& Refractive Surgery, 33, 1851-1854. https://doi.org/10.1016/j.jcrs.2007.07.013

[5] Kwong, Y.Y., Yuen, H.K., Lam, R.F., Lee, V.Y., Rao, S.K., et al. (2007) Comparison of Outcomes of Primary Sclera-Fixated versus Primary Anterior Chamber Intraocular Lens Implantation in Complicated Cataract Surgeries. Ophthalmology, 114, 80-85. https://doi.org/10.1016/j.ophtha.2005.11.024

[6] Singh, O.S. (2000) Nanophthalmos. In: Guidelines for Diagnosis and Therapy, 2nd Edition, Vol. 3, WB Saunders, Philadelphia, 2846-2859.

[7] Hara, S., Borkenstein, A.F.M., Ehmer, A. and Auffarth, G.U. (2011) Retropupillary Fixation of Iris-Claw Intraocular Lens versus Trans Scleral Suturing Fixation for Aphakic Eyes without Capsular Support. Journal of Refractive Surgery, 27, 729-735. https://doi.org/10.3928/1081597X-20110623-01

[8] Kumar, D.A., Agarwal, A., Packiyalakshmi, S., et al. (2013) Complications and Visual Outcomes after Glued Foldable Intraocular Lens Implantation in Eyes with Inadequate Capsules. Journal of Cataract \& Refractive Surgery, 39, 1211-1218. https://doi.org/10.1016/j.jcrs.2013.03.004

[9] Kumar, D.A., Agarwal, A., Agarwal, A., Prakash, G. and Jacob, S. (2011) Glued Intraocular Lens Implantation for Eyes with Defective Capsules: A Retrospective Analysis of Anatomical and Functional Outcome. Saudi Journal of Ophthalmology, 25, 245-254. https://doi.org/10.1016/j.sjopt.2011.04.001

[10] Narang, P. and Narang, S. (2013) Glue-Assisted Intra-Scleral Fixation of Posterior Chamber Intraocular Lens. Indian Journal of Ophthalmology, 61, 163-167. https://doi.org/10.4103/0301-4738.112160

[11] McKee, Y., Price Jr., F.W., Feng, M.T. and Price, M.O. (2014) Implementation of the Posterior Chamber Intraocular Lens Intra-Scleral Haptic Fixation Technique (Glued Intraocular Lens) in a United States Practice: Outcomes and Insights. Journal of Cataract \& Refractive Surgery, 40, 2099-2105.

https://doi.org/10.1016/j.jcrs.2014.04.027

[12] Brandner, M., Thaler-Saliba, S., Plainer, S., Vidic, B., El-Shabrawi, Y., et al. (2015) Retropupillary Fixation of Iris-Claw Intraocular Lens for Aphakic Eyes in Children. PLoS ONE, 10, e0126614. https://doi.org/10.1371/journal.pone.0126614

[13] Lett, K.S. and Chaudhuri, P.R. (2011) Visual Outcomes Following Artisan Aphakia Iris Claw Lens Implantation. Eye (London), 25, 73-76.

https://doi.org/10.1038/eye.2010.146

[14] Rao, R. and Sasidharan, A. (2013) Iris Claw Intraocular Lens: A Viable Option in Monocular Surgical Aphakia. Indian Journal of Ophthalmology, 61, 74.

https://doi.org/10.4103/0301-4738.107198

[15] Menezo, J.L., Martinez, M.C. and Cisneros, A.L. (1996) Iris-Fixated Worst Claw versus Sulcus-Fixated Posterior Chamber Lenses in the Absence of Capsular Support. Journal of Cataract \& Refractive Surgery, 22, 1476-1484. https://doi.org/10.1016/S0886-3350(96)80151-9

[16] Saleh, M., Heitz, A., Bourcier, T., Speeg, C., et al. (2013) Sutureless Intra-Scleral Intraocular Lens Implantation after Ocular Trauma. Journal of Cataract \& Refractive Surgery, 39, 81-86. https://doi.org/10.1016/j.jcrs.2012.08.063

[17] Gonnermann, J., Klamann, M.K., Maier, A.K., Rjasanow, J., Joussen, A.M., et al. (2012) Visual Outcome and Complications after Posterior Iris-Claw Aphakic Intraocular Lens Implantation. Journal of Cataract \& Refractive Surgery, 38, 2139-2143. 
https://doi.org/10.1016/j.jcrs.2012.07.035

[18] Scharioth, G.B., Prasad, S., Georgalas, I., Tataru, C. and Pavlidis, M. (2010) Intermediate Results of Sutureless Intra-Scleral Posterior Chamber Intraocular Lens Fixation. Journal of Cataract \& Refractive Surgery, 36, 254-259.

https://doi.org/10.1016/j.jcrs.2009.09.024

[19] Hazar, L., Kara, N., Bozkurt, E., Ozgurhan, E.B. and Demirok, A. (2013) Intraocular Lens Implantation Procedures in Aphakic Eyes with Insufficient Capsular Support Associated with Previous Cataract Surgery. Journal of Refractive Surgery, 29, 685-691. https://doi.org/10.3928/1081597X-20130723-02

[20] Shanida, H.S., Narayan, S. and Rajini, K.C. (2018) Visual Outcome and Complications of Posterior Iris-Claw Intraocular Lens Implantation: A Follow-Up Analysis. Kerala Journal of Ophthalmology, 30, 107-111.

https://doi.org/10.4103/kjo.kjo_60_18

[21] Ohta, T., Toshida, H. and Murakami, A. (2014) Simplified and Safe Method of Sutureless Intra Scleral Posterior Chamber Intraocular Lens Fixation: Y Fixation Technique. Journal of Cataract \& Refractive Surgery, 40, 2-7.

https://doi.org/10.1016/j.jcrs.2013.11.003

[22] Yamane, S., Inoue, M., Arakawa, A. and Kadonosono, K. (2014) Sutureless 27-Gauge Needle-Guided Intra Scleral Intraocular Lens Implantation with Lamellar Scleral Dissection. Ophthalmology, 121, 61-66. https://doi.org/10.1016/j.ophtha.2013.08.043

[23] Rijneveld, W.J., Beekhuis, W.H., Hassman, E.F., et al. (1994) Iris Claw Lens: Anterior and Posterior Iris Surface Fixation in the Absence of Capsular Support during Penetrating Keratoplasty. Refractive Corneal Surgery, 10, 14-19. 